\title{
G-spot augmentation with autologous fat transplantation
}

\author{
Christian Herold ${ }^{1}$, Melodi Motamedi ${ }^{1}$, Uwe Hartmann², Sixtus Allert ${ }^{1}$ \\ ${ }^{1}$ Department of Plastic and Aesthetic Surgery, Sana Klinikum Hameln Pyrmont, Hameln, Germany \\ ${ }^{2}$ Department of Psychiatry, Social Psychiatry, and Psychotherapy, Division of Psychology, Hannover Medical School, \\ Hannover, Germany
}

\section{Abstract}

Lipofilling for G-spot augmentation is appealing because long-term persistence of the fat is expected to be very good. We report the case of a 29 -year-old patient who requested G-spot augmentation to enhance sexual sensation. Autologous fat (8 cc) that was harvested from the trochanteric area was injected. Although there are few published data acknowledging the presence of the G-spot, the patient was satisfied with the procedure and no side effects occurred. Nevertheless, evaluation with standard questionnaires, such as Fragebogen zur Lebenszufriedenheit (FLZ) and Kurzfragebogen für sexuelle Probleme (KFSP-F), did not indicate the positive effects on subjective well-being and sexual parameters of a surgical G-spot augmentation. Studies comprising a larger series of patients are required before substantiated recommendations regarding the benefits and risks of this procedure will be possible. (J Turk Ger Gynecol Assoc 2015; 16: 187-8)

Keywords: G-spot, fat transplantation, FLZ, KFSP-F, questionnaire

Received: 06 February, 2015

Accepted: 20 May, 2015

Available Online Date: 14 July, 2015

\section{Introduction}

The G-spot had been described more than 60 years ago by the German gynecologist Gräfenberg (1). This area on the anterior wall of the vagina is $10-20 \mathrm{~mm}$ in diameter and is said to be approximately $5 \mathrm{~cm}$ above the ostium of the urethra. In some women, this area is highly sensitive and stimulation may quickly lead to orgasm (2). Although anatomical and biochemical studies have failed to provide evidence regarding the G-spot and only case studies and anecdotal observations support its presence (3), the demand for surgical G-spot augmentation is increasing. Hyaluronic acid or autologous fat are the described fillers for this procedure (4).

\section{Case Presentation}

We report the case of a 29-year-old patient who requested Gspot augmentation to enhance sexual sensation. This patient also consulted our clinic for augmentation/mastopexy and thigh lift as well as for liposuction of the trochanteric region. Therefore, it was decided to use autologous fat in this patient. After informed consent was obtained from the patient, she was administered general anesthesia and was operated in the supine position with straddled legs. Fat graft was harvested by the water-assisted liposuction technique (Body Jet ${ }^{\circledR}$ and LipoCollector $3{ }^{\circledR}$ System, Human Med, Schwerin, Germany). The urethra was secured with a catheter that also stabilized the anterior vaginal wall and made the injection technique simpler. After insertion of a speculum, an injection of 8-cc fat graft (Figure 1) was administered strictly in the submucosal layer $5 \mathrm{~cm}$ away from the introitus of the urethra, taking care not to injure the urethra and bladder and not to perforate the vaginal wall a second time because this would result in the fat graft leaking from the injection site. A 18-G sharp needle (BD, Franklin Lakes, NJ, USA) and a 5-cc syringe (BD, Franklin Lakes, NJ, USA) were used. No sutures or dressings were used, and the patient was recommended not to have sexual intercourse for 1 week following the surgery. The postoperative course was uneventful, and the patient did not report any pain at the injection site.

Postoperatively, the patient presented to our clinic for followup and reported an initial increased ability to stimulate the amplified G-spot during the first 2 weeks that normalized after the first 2 weeks. The patient was asked to complete the Fragebogen zur Lebenszufriedenheit (FLZ) questionnaire, a standard tool for the evaluation of satisfaction and happiness in life, 1 and 20 months postoperatively. The item Spouse/Partner was rated preoperatively and one month postoperatively as "very happy," stanine 9 , meaning within the highest $4 \%$ of the representative sample for normative comparison, and stanine 5, meaning average satisfaction after 20 months. The item Sexuality was rated preoperatively and postoperatively at all time points as "rather unhappy," stanine 3, meaning within the lowest $23 \%$ of the representative sample for normative comparison. The item Self was rated preoperatively and postoperatively after one month "rather unhappy," stanine 3, meaning within the lowest $23 \%$ of the representative sample for normative comparison, but stanine 5, meaning average satisfaction after 20 months. 


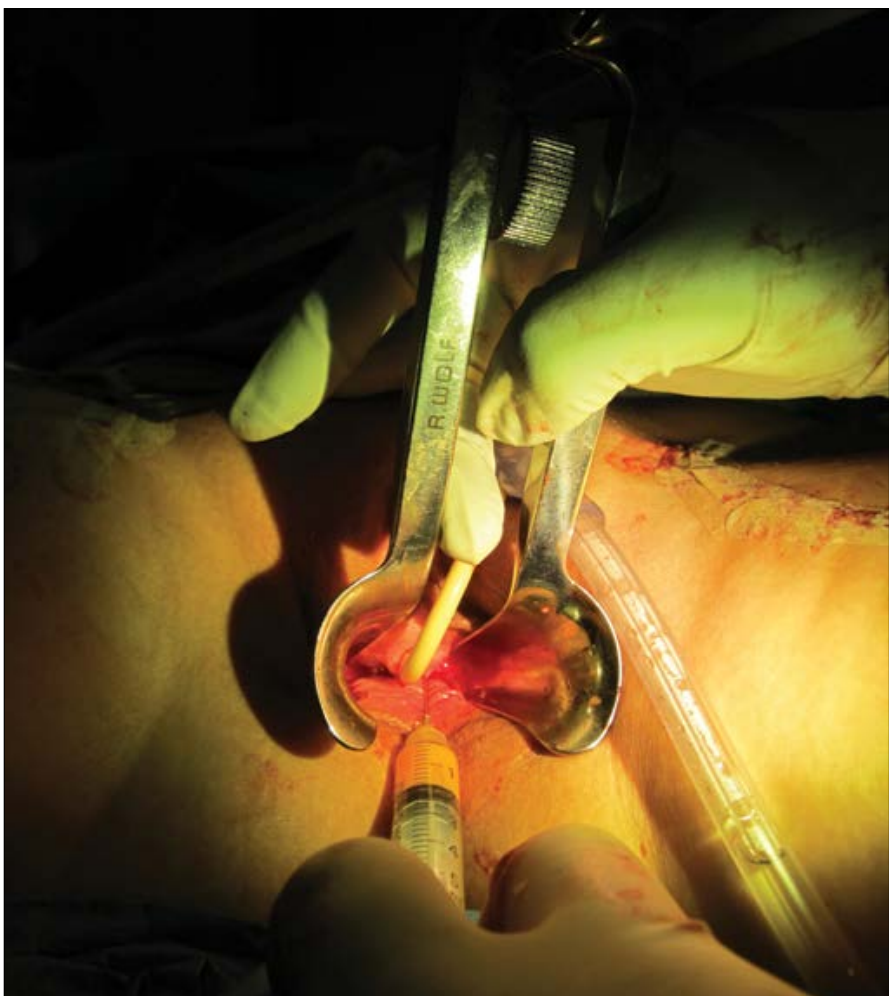

Figure 1. After insertion of a speculum, a strict submucosal injection of 8-cc fat graft was administered using a 18-G needle and a 5-cc syringe in the area of the G-spot $5 \mathrm{~cm}$ away from the introitus of the urethra, taking care not to injure the urethra and bladder

The Kurzfragebogen für sexuelle Probleme (KFSP-F) questionnaire is a German adaptation of the brief index of sexual functioning for women (5). This 28-item questionnaire was employed to assess problems in five major dimensions of female sexuality (desire, arousal, orgasm, sexual pain, and sexual satisfaction). KFSP-F as well as FLT questionnaires have been validated for people living in Germany. Nevertheless, they have not been explicitly validated for Turkish women. KFSP-F was applied preoperatively and postoperatively. Postoperatively at both time points, the patient reported a greater importance of a satisfying sexual life and a stronger sexual desire as well as a higher willingness to initiate sexual activity. Nevertheless, she was not able to reach orgasm during vaginal sexual intercourse neither before nor after G-spot amplification. At all time points, auto stimulation was the only way for the patient to reach orgasm.

\section{Discussion}

The evidence to support the presence of the G-spot is weak (3). Published scientific data highlight the fact that the G-spot does not even exist. In a recent review, Puppo et al. (6) proposed that G-spot amplification is an unnecessary and inefficacious procedure. Nevertheless, introital injections of hyaluronic acid have been able to increase sexual satisfaction $(4,7)$. It is unclear whether these finding are based on the anatomical changes or rather based on imagination of the patients treated. Autologous fat transplantation has also been used to amplify the G-spot area. In a book article, Gress (4) describes an amelioration of sexual stimulation after autologous fat transplantation in $52 \%$ of his patients. In these patients, 5-12-cc fat graft had been injected. Lipofilling to amplify the G-spot area is appealing because long-term persistence of the fat is expected to be very good. Exact volumetric measurements are missing; however, because of the very good vascularity of the vaginal mucosa, volume survival rates should be superior to the breast tissue, where survival rates of $>70 \%$ have been demonstrated using magnetic resonance imaging volumetry (8).

Despite all positive preliminary results (4), patients seeking Gspot augmentation should be thoroughly informed as dyspareunia, infection, or scarring are possible complications (9).

To the best of our knowledge, the effect of G-spot amplification has not been previously investigated by applying standard questionnaires. The results of this case report did not indicate positive effects on subjective well-being and sexual parameters of a surgical G-spot augmentation. A larger series of patients is required before substantiated recommendations regarding the possible benefits and risks of this procedure will be possible; the scientific evaluation of the presented case at least fails to prove any efficiency.

Ethics Committee Approval: N/A.

Informed Consent: Written informed consent was obtained from patient who participated in this case.

Peer-review: Externally peer-reviewed.

Author Contributions: Concept - C.H., M.M., S.A.; Design - C.H., S.A.; Supervision - S.A., U.H.; Resource - S.A.; Materials - M.M., C.H., S.A., U.H.; Data Collection and/or Processing - M.M., C.H., S.A., U.H.; Analysis and/or Interpretation - M.M., C.H., S.A., U.H.; Literature Search - M.M., C.H., U.H.; Writing - M.M., C.H. S.A., U.H.; Critical Reviews - M.M., C.H., S.A., U.H.

Conflict of Interest: No conflict of interest was declared by the authors.

Financial Disclosure: The authors declared that this study has received no financial support.

\section{References}

1. Gräfenberg E. The role of urethra in female orgasm. Int J Sexol $1950 ; 145-8$.

2. Gravina GL, Brandetti F, Martini P, Carosa E, Di Stasi SM, Morano S, et al. Measurement of the thickness of the urethrovaginal space in women with or without vaginal orgasm. J Sex Med 2008; 5: 610-8. [CrossRef]

3. Hines TM. The G-spot: a modern gynecologic myth. Am J Obstet Gynecol 2001; 185: 359-62. [CrossRef]

4. Gress S. Form- und funktionsverbessernde Eingriffe im weiblichen Genitalbereich. In: Ästhetische Chirurgie von Heimburg Lemperle 2012; 25.

5. Mazer NA, Leiblum SR, Rosen RC. The brief Index of sexual Functioning for women (BIFS-W): a new scoring algorithm and comparison of normative and surgically menopausal populations. Menopause 2000; 5: 350-63. [CrossRef]

6. Puppo V, Gruenwald I. Does the G-spot exist? A review of the current literature. Int Urogynecol J 2012; 23: 1665-9. [CrossRef]

7. Andrighetti $\mathrm{S}$, Baker KA. A pioneering nonsurgical alternative for vaginal rejuvanation using hyaluronic acid volumizing filler (Juvederm Voluma). J Sex Med 2012; 9: 287.

8. Herold C, Ueberreiter K, Cromme F, Busche MN, Vogt PM. The use of mamma MRI volumetry to evaluate the rate of fat survival after autologous lipotransfer. Handchir Mikrochir Plast Chir 2010; 42: 129-34. [CrossRef]

9. Committee on Gynecologic Practice, American College of Obstetricians and Gynecologists. ACOG Committee Opinion No. 378: Vaginal "rejuvenation" and cosmetic vaginal procedures. Obstet Gynecol 2007; 110: 737-8. [CrossRef] 